Recepción: 21/ 07 / 2018

Aceptación: 13 / 09 / 2018

Publicación: 01 / 11 / 2018

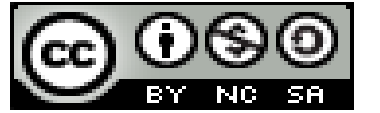

Ciencias económicas y empresariales Artículo de investigación

\title{
Control interno en los costos de fabricación de los productos lácteos en la Cooperativa de Producción Agropecuaria "Chone Ltda"
}

Internal control in the costs of manufacturing dairy products in the Agricultural Production Cooperative "Chone Ltda."

Controle interno nos custos de fabricação de produtos lácteos na Cooperativa de Produção Agrícola "Chone Ltda"

\author{
Susy T. Toala-Mendoza ${ }^{\mathrm{I}}$ \\ susygemma-19@hotmail.com \\ Karina C. Arteaga Muñoz II \\ karina.arteaga@uleam.edu.ec \\ Jhon A. Álava-Intriago III \\ arturo.alava@uleam.edu.ec
}

Correspondencia: susygemma-19@hotmail.com

\footnotetext{
${ }^{\text {I }}$ Magíster en Auditoria Integral, Ingeniera en Contabilidad y Auditoría, Docente de la Escuela Superior Politécnica de Manabí Manuel Félix López, Manta, Ecuador.

${ }^{\text {II }}$ Docente de la Universidad Laica Eloy Alfaro de Manabí, Manta, Ecuador.

III Docente de la Universidad Laica Eloy Alfaro de Manabí, Manta, Ecuador.
} 


\title{
Resumen
}

This research work consists of carrying out an analysis of the accounting processes that are executed in the Cooperative of Agricultural Production of Chone Ltda, through the application of the internal control system and thus be able to identify which are the strengths and weaknesses of the company. The same will allow to know what are the errors that should improve and change, so that this entity is a leader in the market in the processing of dairy products. In order to achieve better control, it was necessary to elaborate a procedure manual for the internal accounting control system, obtaining transparent information that helps decision-making preventing future risk.

In order to achieve the stated objective, the analysis methods were used, and the techniques as a support tool for interviews and surveys, which are aimed at the manager and the employees of the company. The present work is structured in three chapters detailing each process; in the first CHAPTER, it is the fundamental part where the theoretical framework is described investigating a scientific study focused on the theories (content, importance, characteristic, objectives, elements, among others), which complements the work of the research, fulfilling the objectives proposed.

In the second CHAPTER where the study carried out in the research field is described, tabulating data by means of statistical graphs on the current situation of the Cooperativa Agropecuaria de Chone Ltda. Applying the basic techniques such as the interview and survey to verify the hypothesis presented in the present work.

In the third CHAPTER proceeds to develop the manual of procedures to the internal accounting control system as a proposal for the Agricultural Cooperative which will improve internal processes in the development of daily activities in the development of a product.

Palabras clave: manual; procedures; system; internal control; accounting processes.

\begin{abstract}
This research work consists of carrying out an analysis of the accounting processes that are executed in the Cooperative of Agricultural Production of Chone Ltda, through the application of the internal control system and thus able to identify which are the strengths and weaknesses of the company. The same will allow to know what are the errors that should improve and change, so that this entity is a leader in the market in the processing of dairy products. In order to achieve
\end{abstract}


better control, it was necessary to elaborate a manual for the internal accounting control system, obtaining transparent information that helps decision-making preventing future risk.

In order to achieve the stated objective, the analysis methods were used, and the techniques as a support tool for interviews and surveys, which are aimed at the manager and the employees of the company. The present work is structured in three chapters detailing each process; in the first CHAPTER, it is the fundamental part where the theoretical framework is described investigating a scientific study focused on the theories (content, importance, characteristic, objectives, elements, among others), which complements the work of the research, fulfilling the objectives proposed.

In the second CHAPTER where the study carried out in the research field is described, tabulating data by means of statistical graphs on the current situation of the Cooperativa Agropecuaria de Chone Ltda. Applying the basic techniques such as the interview and survey to verify the hypothesis presented in the present work.

In the third CHAPTER proceeds to develop the manual of procedures to the internal accounting control system as a proposal for the Agricultural Cooperative which will improve internal processes in the development of daily activities in the development of a product.

Key words: manual; procedures; system; internal control; accounting processes.

\section{Resumo}

Este trabalho de pesquisa Consistem de realizar uma análise dos processos de contabilidade que são executados na Cooperativa da Produção Agrícola de Chone Ltda, através da aplicação do sistema de controlo interno e, assim, ser capaz de identificar quais são os pontos fortes e fracos da empresa. O mesmo permitirá saber quais são os erros que devem melhorar e mudar, para que esta entidade seja líder no mercado no processamento de produtos lácteos. Para obter um melhor controle, foi necessário elaborar um manual para o sistema de controle contábil interno, obtendo informações transparentes que auxiliam na tomada de decisões, prevenindo riscos futuros.

Para atingir o objetivo declarado, foram utilizados os métodos de análise e as técnicas como ferramenta de apoio a entrevistas e pesquisas, voltadas ao gestor e aos funcionários da empresa. O presente trabalho está estruturado em três capítulos detalhando cada processo; no primeiro capítulo, é essencial a parte onde o quadro teórico é descrito investigando um estudo científico 
focado nas teorias (conteúdo, importância, característica, Objetivos, elementos, entre outros), que complementa o trabalho da pesquisa, cumprindo os Objetivos proposto.

No segundo capítulo onde o estudo realizado no campo da pesquisa é descrito, tabulação de dados por meio de gráficos estatísticos sobre a situação atual da Cooperativa Agrícola Chone Ltda Aplicando as técnicas básicas: Tais como a entrevista e pesquisa para verificar a hipótese apresentada no presente trabalho.

No terceiro capítulo procede-se a desenvolver o manual de procedimentos para o sistema de controle contábil interno como uma proposta para a Cooperativa Agrícola que melhorará os processos internos no desenvolvimento das atividades diárias no desenvolvimento de um produto. Palavras chave: manual; procedimentos; sistema; controle interno; processos contábeis.

\section{Introducción}

El control interno es el componente más importante en la parte interna de una empresa, estabilizando los procesos de las actividades diarias para financiar los proyectos planteados a futuro, logrando un mejor control en las actividades de cada departamento conforme está dividida la organización, la Cooperativa de Producción Agropecuaria Chone Ltda. pretende mejorar los costos de fabricación en los productos lácteos, el propósito del presente trabajo de investigación es evaluar, es la aplicación de un control interno en los procesos de producción para mejorar los precios adecuados para el consumidor final.

Estabilizar costos no afectaría la calidad del producto que la cooperativa ofrece, más bien ayudaría a sistematizar los recursos que la misma determina para alcanzar el objetivo planteado, con el fin de mejorar la utilidad logrando manejar y controlar eficazmente los costos. También se busca determinar si existen deficiencias en los procesos de elaboración en los productos lácteos, así como poner en práctica las posibles soluciones del buen control interno dentro de la cooperativa agropecuaria Chone Ltda. Por lo tanto, la realización de la investigación permitirá que las actividades del área estén en manos de personal idóneo, y los directivos de la cooperativa puedan tomar mejores decisiones que contenga como resultado beneficio a futuro, esto es con la finalidad de tener más vigilancia y mejor control en las operaciones que se realizan. 
Las aplicaciones del control interno en los costos de fabricación son principalmente para determinar la efectividad y eficiencia en el uso de los recursos que se mantienen en la Cooperativa Agropecuaria de Chone y el impacto socio- económico derivados de sus actividades y procesos, cabe reconocer que este proceso de investigación ayudará a mejorar en las buenas prácticas clasificando los recursos necesario sean directos e indirectos, incluyendo los costos fijos y costos variable, evaluar los costos derivados de la misma estandarizando el nivel de venta, mejorando el rendimiento económico de la cooperativa permitiendo obtener más ingresos $\mathrm{y}$ tomar decisiones que puedan mejorar la estabilidad en el mercado actual.

Carrillo, (2014) manifiesta que el Control Interno es un proceso integral efectuado por el titular, funcionario y servidores de una entidad, diseñado para enfrentar los riesgos y para dar seguridad razonable de que, en la consecución de la misión de la entidad, se alcanzaran los objetivos de la misma, es decir, es la gestión misma orientada a minimizar los riesgos.

Con la aplicación del control interno optimizará los recursos obtenidos en el proceso de producción minimizando riesgos que pueden surgir en la operación de productos lácteos, con fines de mejorar la rentabilidad económica de la inversión logrando los objetivos establecidos en los proyectos realizados.

El control interno es el conjunto de procedimientos, políticas, directrices y planes de organización los cuales tienen por objeto asegurar una eficiente, seguridad y orden en la gestión financiera, contable y administrativa de la empresa; salvaguardia de los activos, fidelidad del proceso de información y registros, cumplimientos de políticas definidas (Barrios, 2014).

Para una organización el control interno consiste en ajustar el criterio de control seleccionado a las necesidades en características particulares de dicha organización y diseñado para promocionar seguridad razonable. Un buen control interno en la cooperativa ayudará a los directivos a obtener mejores resultados en las operaciones diarias y por ende a los miembros facilitar el proceso de producción teniendo claro los objetivos a realizar. Mediante registros ordenados por las operaciones realizadas en la producción, almacenando información adecuada con eficiencia y eficacia en la administración de la cooperativa tomando decisiones con visión productiva. 


\section{Control interno}

El control interno se origina después del nacimiento de la contabilidad mediante el manejo de la partida doble, y posterior a ello en la revolución industrial es cuando surge la necesidad de controlar las operaciones que por su magnitud eran realizadas por máquinas manejadas por varias personas. Según Catácora (1996), el control interno "Es la base sobre el cual descansa la confiabilidad de un sistema contable, el grado de fortaleza determinará si existe una seguridad razonable de las operaciones reflejadas en los estados financieros."

El control interno también se define como "Un proceso efectuado por el consejo de administración, la dirección y el resto del personal de una entidad, diseñado con el objeto de proporcionar un grado de seguridad razonable en cuanto a la consecución de objetivos dentro de las siguientes categorías:

Honestidad y responsabilidad,

Eficiencia y eficacia de las operaciones,

Fiabilidad de la información,

Salvaguarda de los recursos; $y$,

Cumplimiento de las leyes y norma.

El control interno ha iniciado su primera definición a través de un informe denominado COSO, (Committee of Sponsoring Organizations of the Treadway Comisión) el mismo que expresa su opinión acerca de la situación financiera de una entidad, a su vez permitiendo la implementación de un adecuado método para un mejor manejo de todas sus operaciones dentro de la misma, (Gómez, Blanco, \& Conde, 2013). En las últimas décadas está enfocado prioritariamente a promover con eficiencia, eficacia cada uno de los procesos cumpliendo con las políticas establecidas. Es un proceso efectuado por la máxima autoridad y el

resto del personal, cuya finalidad es obtener una seguridad razonable de todas las actividades dentro de la empresa. Con el fin de salvaguardar todos los activos y a su vez permitir la obtención de información financiera correcta, oportuna y segura. (Nagua Cartuche, 2016). 
"El control interno es aquel que hace referencia al conjunto de procedimientos de verificación automática que se producen por la coincidencia de los datos reportados por diversos departamentos o centros operativos." En sentido estricto "El control interno es una expresión utilizada para describir todas las medidas tomadas por los propietarios y directores de empresas para dirigir y controlar a los empleados" En sentido amplio "Conjunto de elementos, normas y procedimientos a lograr, a través de una efectiva planeación, ejecución y control, el ejercicio eficiente de la gestión, para el logro de los fines de la organización”

El control es un factor clave en lo logro de los objetivos generales de las organizaciones por ello debe ser oportuno, económico, seguir una estructura orgánica, debe tener una ubicación estratégica, revelar tendencias y situaciones. En la vida real, un gran volumen de las decisiones administrativas está basado en información de tipo contable.

\section{Importancia del control interno}

Radica en que permiten a la gerencia hacer frente a la rápida evolución del entorno económico y competitivo, así como a las exigencias y prioridades cambiantes de los clientes, adaptando su estructura para asegurar el crecimiento futuro. (Rivas Márquez, 2011)

\section{El control interno es de vital importancia dado que:}

Establece medidas para corregir las actividades, de tal forma que se alcancen planes exitosamente.

Se aplica a todo (cosas, personas y a los actos.)

Determina y analiza rápidamente las causas que pueden originar desviaciones, para que no se vuelvan a presentar en el futuro.

Localiza a los lectores responsables de la administración, desde el momento que se establecen medidas correctivas.

Proporciona información acerca de la situación de la ejecución de los planes, sirviendo como fundamento al reiniciarse el proceso de planeación.

Reduce costos y ahorra tiempo al evitar errores. 
$\mathrm{Su}$ aplicación incide directamente en la racionalización de la administración y consecuentemente, en el logro de la productividad de todos los recursos de la empresa. (Quiroz, 2011)

\section{Objetivos del control interno}

Lo que se espera del Control Interno es que brinde la mayor seguridad para la consecución de los objetivos, a través de la correcta aplicación de los reglamentos, políticas y procedimientos establecidos por la alta dirección, quien a su vez debe actuar como un supervisor para que dichos lineamientos sean cumplidos.

\section{Entre los objetivos del Control Interno podemos mencionar:}

Salvaguardar los activos de la organización evitando pérdidas por fraudes o negligencias.

Asegurar la exactitud y veracidad de los datos contables y financieros, que son utilizados por la dirección para una adecuada toma de decisiones.

Incentivar la eficiencia en el uso de los recursos.

Estimular el seguimiento de las prácticas decretadas por la gerencia

Promover, evaluar y velar por la seguridad, calidad la mejora continua de todos los procesos en general.

Adoptar medidas de protección para los activos físicos en forma adecuada.

Determinar lineamientos para evitar que se realicen procesos sin las adecuadas autorizaciones

Generar una cultura de control en todos los niveles de la organización. (Rodrigo, 2006)

Cada entidad fija su misión, estableciendo los objetivos que espera alcanzar y las estrategias para conseguirlos, los objetivos pueden ser para la entidad como

un todo o específicos para las actividades dentro de la entidad, aunque muchos objetivos pueden ser específicos para una entidad particular, algunos son ampliamente participativos.

Operaciones, relacionadas con el uso efectivo y eficiente de los recursos de la entidad. 
Información Financiera, relacionada con la preparación de estados financieros públicos confiables.

Cumplimiento, relacionado con el cumplimiento de la entidad con las leyes y regulaciones aplicables.

El autor Rodrigo estupiñan Gaitan establece los objetivos de la siguiente manera, "El control interno comprende el plan de la organización y el conjunto de métodos y procedimientos, que aseguren que los activos están debidamente protegidos que los registros contables son fidedignos y que la actividad de la entidad se desarrolla eficazmente según las directrices marcadas por la administración."

\section{De acuerdo a lo anterior los objetivos básicos son:}

Proteger los activos y salvaguardar los bienes de la institución.

Verificar la razonabilidad y la confiabilidad de los informes contables y administrativa.

Promover la adhesión a las políticas administrativas establecida.

Lograr el cumplimiento de las metas y objetivos programados.

Beneficios del control interno

\section{Los beneficios incluyen:}

Ayudar a los directivos al logro razonable de las metas y objetivos institucionales Integrar e involucrar al personal con los objetivos de control

Ayudar al personal a medir su desempeño y, por ende, a mejorarlo

Contribuir a evitar el fraude

Facilitar a los directivos la información de cómo se han aplicado los recursos y cómo se han alcanzado los objetivos.

Elementos y principios básicos de control interno 


\section{Partes constituyentes de un sistema de control interno son:}

Una variable, es el elemento (o programa objetivo) que se desea controlar (características a ser controladas).

Mecanismos sensores, que son sensibles para medir las variaciones o los cambios de las variables.

Medios Motores, a través de los cuales se puede desarrollar las acciones correctivas.

Fuentes de Energía, que entrega la energía (o información) necesaria para cualquier tipo de actividad.

Retroalimentación, mediante la cual, a través de la comunicación del estado de la variable por los sensores, se logran llevar a cabo las acciones correctivas.

\section{El autor Milton Maldonado en su obra El Control Interno, sugiere que los elementos básicos en un Sistema de Control Interno son los siguientes:}

Un Plan de organización que prevea una separación apropiada de responsabilidades funcionales

Un sistema de autorización y procedimientos de registros adecuados para proveer un control financiero razonable sobre los activos, pasivos, ingresos y gastos

El Sistema de Control Interno y el aseguramiento de la calidad,

Un grado de idoneidad del personal proporcional a sus responsabilidades

(entrenamiento, eficiencia, moralidad, retribución)

Una función efectiva de auditoria interna.

Clasificación del control interno 
Los controles de la organización de una manera convencional pueden clasificarse de cuatro formas:

\section{Según su relación con el controlado:}

Control interno: se efectúa desde las funciones propias de la organización. Ejemplo Control de asistencia por parte de la Gerencia de Recursos Humanos.

Control externo: se realiza por agentes externos de la organización.

Ejemplo: Control del Municipio sobre las disposiciones de desechos contaminantes.

Control directo: los controles se pueden identificar claramente o están diseñados específicamente al proceso, al producto o a la persona. Ejemplo pruebas de resistencias de las cuerdas de material sintético (al producto)

Control indirecto: el efecto del sensor llega de forma indirecta a otros elementos controlados. Ejemplo: Medición de la temperatura ambiente (afecta a las condiciones de la materia prima, productos en proceso y en terminados, como también a las personas).

\section{Según la oportunidad de realización:}

Control perceptivo o concurrente: el que se produce al momento en que se está ejecutando un acto administrativo. Ejemplo: El control de arena, ripio, y cemento se mezclan al momento de una fundición.

Control posterior: el que se realiza después de que se ha efectuado una actividad. Dentro de este control se encuentra la auditoria. Ejemplo verificación de la calidad de las losas de una edificación.

Según su alcance: aplican a la presencia de las personas en la organización. Ejemplo control

Sobre los procesos: a al funcionamiento de los procesos. Ejemplo controles al proceso de empaquetamiento de productos terminados.

Sobre la gestión: aplican al control de gestión o a la administración de los recursos empresariales y la obtención de objetivos. Ejemplo: evaluación del desempeño en cuanto al cumplimiento de las metas y logros semanales. 


\section{Por la función:}

Control administrativo: Aplica a todas las operaciones de la organización.

Ejemplo: Controles de asistencia al personal.

Control financiero: aplica a las operaciones que involucran recursos financieros. Ejemplo Controles de manejo de fondos en cuentas corrientes.

Control técnico: aplican las actividades técnicas relacionadas al giro del negocio. Ejemplo Mantenimiento de maquinaria especializada.

Control jurídico: aplica a los aspectos jurídicos que afectan a la gestión.

Ejemplo: seguimiento al patrocinio de las causas en las que actúa la organización como demandante o actor. (Quiroz, 2011)

\section{Sistema de Control Interno}

"Comprende el plan de organización y el conjunto coordinado de métodos y medidas adoptadas dentro de una empresa para salvaguardar sus activos, verificar la exactitud y confiabilidad de la información contable, promover la eficiencia operativa y alentar la adhesión a las políticas prescriptas por la Dirección Superior”. (Moncada, 2008)

El sistema de control interno es preciso en las empresas ya que por medio de este sistema se puede realizar planes, técnicas, normas, procedimientos que permitan verificar y evaluar a la entidad como se encuentra. Con el fin de que todas las actividades realizadas sean seguras, los activos sean salvaguardados, obtengan una información confiable, cumplan con todas las normas y leyes vigentes establecidas dentro de las políticas marcadas por la alta dirección.

Un sistema de control interno es muy significativo porque permite planear y nunca dejar a la casualidad, ya que dispone de métodos de cómo debe funcionar la administración de la empresa. Es decir, coordinadamente con eficacia y seguridad, de manera que garanticen máxima seguridad y control de los recursos. 


\section{Componentes del Control Interno.}

El control Interno consta de cinco componentes cada uno de ellos constituye el órgano fundamental de toda organización, permitiendo un adecuado y correcto funcionamiento dentro de la misma, la cuales son:

El ambiente de control.

Evaluación de riegos.

Actividades de control

Información y comunicación

Monitoreo. (Nagua Cartuche, 2016).

De acuerdo al marco integrado de control interno COSO (Modelo COSO)

(Rivas Márquez, 2011) define el control interno que consta de cinco categorías o componentes que la administración diseña y aplica para proporcionar una seguridad razonable de que sus objetivos de control se llevaran a cabo de manera adecuada. Estos componentes son:

Primer componente: Ambiente de Control. - Ambiente de control de una empresa, es la actitud general de sus administradores y empleados hacia la importancia del control interno.

Segundo componente: Evaluación de los Riesgos. - La evaluación de los riesgos sirve para describir el proceso con que los ejecutivos identifican, analizan y administran los riesgos de negocio que puede enfrentar una empresa y el resultado de ello.

Tercer Componente: Actividades de Control. - Son las políticas y procedimientos que ayudan a asegurar que se están llevando a cabo las directrices administrativas. Se establecen con el propósito de garantizar que las metas de la empresa se alcancen. Las actividades de control consideradas en la estructura conceptual integrada COSO son las siguientes:

Revisiones de alto nivel. - Incluye la comparación del desempeño contra presupuestos, pronósticos, etc. Procesamiento de la información, se realiza una variedad de controles a fin de verificar la precisión, integridad y autorización de las transacciones. 
Funciones directas o actividades administrativas. - Los administradores dirigen las funciones o las actividades revisando informes de desempeño.

Controles físicos. - Equipos, inventarios y otros activos se aseguran físicamente en forma periódica son contados y comparados con las cantidades presentadas en los registros de control. Indicadores de desempeño, relacionar unos con otros los diferentes conjuntos de datos operacionales.

Indicadores de desempeño. - Relacionar unos con otros los diferentes conjuntos de datos operacionales o financieros, además de analizar las interrelaciones e investigar y corregir las acciones.

Segregación de responsabilidades. - Para reducir el riesgo de error o de acciones inapropiadas. Para ello existen cuatro guías generales que orientan las segregaciones adecuadas de las responsabilidades:

Contabilidad separada de la custodia de los activos financieros;

Custodia de activos relacionados separados de la autorización de operaciones;

Responsabilidad operativa separada de la responsabilidad de registro contable;

Deberes y responsabilidades del departamento de tecnología de información separado de los departamentos de usuarios.

Cuarto componente: Información y comunicación. - La información y la comunicación son elementos esenciales en una estructura de control interno. La información acerca del ambiente de control, la evaluación de los riesgos y los procedimientos de control y la supervisión son necesarios para que los administradores puedan dirigir las operaciones y garantizar que sean puesto en práctica las normativas legales, reglamentarias y de información.

Este componente de control interno, se refiere a los métodos empleados para identificar, reunir, clasificar, registrar e informar acerca de las operaciones de la entidad y para conservar la contabilidad de los activos relacionados.

Quinto componente: Monitoreo. - Las actividades de monitoreo se refieren a la evaluación continua o periódica de calidad del desempeño del control interno, con el propósito de determinar 
qué controles están operando de acuerdo con lo planeado y que se modifiquen según los cambios en las condiciones. (Rivas Márquez, 2011)

El control interno en el ambiente empresarial.

El entorno económico, político y social generan cambios en los sistemas de control interno de las organizaciones empresariales ya que los riesgos varían, las necesidades son otras, las culturas se transforman. (Gaitan, 2015)

Costo de Fabricación.

\section{Definición}

El costo de producción o costo del producto es el resultado de sumar el valor de los materiales consumidos (material directo), los salarios pagados a los operarios de producción (mano de obra directa) y la suma de los pagos realizados o causados correspondientes a todas las actividades adicionales necesarias para elaborar ese producto o servicio (costos indirectos de fabricación). Independientemente del sistema de costeo utilizado, la distribución adecuada de los costos indirectos de fabricación (CIF), repercute directamente en el valor asignado al producto, por esta razón se debe prestar la debida atención al procedimiento de asignación realizado. (Arias Montoya, Portilla, \& Fernández,

2010)

"Costo es todo consumo o egreso causado en el área de operaciones o producción, que agrega valor al servicio o producto final y que se puede activar en calidad de inventario." (Bonilla y Prada, 1999). Para Reyes (s.f.) es el conjunto de recursos que intervienen en el proceso productivo en un período determinado y que son captados, registrados y valorados según los criterios de la Contabilidad de Costos y Amaro (2002) lo presenta como el valor del stock de bienes de cambio de la contabilidad patrimonial. (Aizaga, 2016)

Según el Diccionario de la Contaduría General de la Nación (1998) los Costos son erogaciones o causaciones de obligaciones ciertas, relacionadas directamente con los procesos de producción de bienes o prestación de servicios que se recuperan en el desarrollo de la actividad de enajenación. En su acepción más simple y contablemente hablando, Hongren et al. (1996) y 
Polimeni et al. (1994), definen un costo como un recurso al que se sacrifica o al que se renuncia para alcanzar un objetivo específico. Los costos se miden en unidades monetarias y constituyen el valor que se paga para adquirir bienes y servicios.

Por ende, los costos de producción en cualquier organización requieren de un tratamiento especial para lograr mantener la empresa en el mercado; éstos deben ser monitoreados constantemente para garantizar la rentabilidad y la ganancia neta de las empresas, que de esta manera pueden ofrecer productos con precios competitivos en el mercado.

En tal sentido, al definir los costos de producción (también llamados costos de fabricación o manufactura) se agregan nuevos elementos; los desembolsos necesarios para mantener un proyecto, línea de procesamiento o un equipo en funcionamiento.

Según el Diccionario de la Contaduría General de la Nación (1998), un costo de producción es una denominación que agrupa las cuentas representativas de las erogaciones asociadas directamente con la elaboración o producción de bienes, o la prestación de servicios de los cuales se obtienen los ingresos.

\section{Características}

El costo de producción tiene dos características opuestas, que algunas veces no son bien entendidas en los países en vías de desarrollo; la primera es que para producir bienes se debe generar un costo;

La segunda es que los costos deberían ser mantenidos tan bajos como sea posible y/o eliminar los innecesarios.

\section{Clasificación de los costó de fabricación}

Los costos de materiales. - Están relacionados con los principales recursos que se usan en la producción. Los costos de materiales, son aquellos que se transforman en bienes terminados con la adición de mano de obra directa y costos indirectos de fabricación.

Los costos de mano de obra. - Se refieren al esfuerzo físico o mental empleado en la fabricación de un producto. Los costos de mano de obra pueden dividirse en mano de obra directa e indirecta. 
Los Costos de Mano de obra directa. - Se refieren a las compensaciones de toda la mano de obra directamente involucrada en la fabricación de un producto terminado y puede asociarse a éste con facilidad.

Mano de obra indirecta. - Se refiere a aquella involucrada en la fabricación de un producto que no se considera mano de obra directa, por lo cual se incluye como parte de los costos indirectos de fabricación.

Los costos indirectos de fabricación. - Son aquellos que, aunque se consideran como parte del objeto de costos, no puede realizarse un seguimiento en forma económicamente factible. Esta categoría de costos se utiliza para acumular los materiales indirectos, la mano de obra indirecta y los demás costos indirectos de fabricación que no pueden identificarse directamente con los productos específicos. (Orozco, 2013)

Juárez, Rodríguez, López, López y Arenas (2006) refieren costos basados en el volumen y en el tiempo, se relacionan a continuación las:

\section{Clasificaciones más comunes:}

Sistema de costos por órdenes y por procesos.

Costos por absorción o inventariarles. - Método por el cual los costos indirectos de fabricación fijos forman parte del valor de los inventarios.

Costos directos o variables. - Se excluyen de los inventariarles.

Costos por actividades (Brinson, 1997). - Identifican las actividades en

una organización y determinan su costo y rendimiento (tiempo y calidad).

\section{Reyes (s.f.) dispone la siguiente clasificación:}

De acuerdo con la forma como se expresen los datos. - según la fecha y el método de cálculo. Se tienen los siguientes sistemas de costos.

Históricos. - Son los que asignan a los productos fabricados todos los costos reales en que se incurre durante un período determinado de tiempo en la fabricación de éstos.

Costos predeterminados. - Son costos que se calculan antes de que se 
inicie la producción. Cuando se usa un sistema de contabilidad de costos predeterminados, tanto los costos predeterminados como los reales se reflejan en la contabilidad.

\section{De acuerdo a su Variabilidad}

Los costos no solo relacionan de manera directa o indirecta con los trabajos, productos o procesos, sino que también pueden variar en cantidad con los cambios en la producción. Es así, como se tienen:

Costos Variables. - Son aquellos costos que tienden a aumentar o disminuir en total, en proporción con los cambios en los niveles de actividad ejemplo: mano de obra directa, materia prima directa y gastos de fabricación variables. Estos costos en términos unitarios son constantes.

Costos fijos. - Son aquellos costos que no varían dentro de un nivel normal de operaciones y dado un cierto período de tiempo. Ejemplo: sueldos, arriendos, etc.

\section{De acuerdo a la naturaleza de las operaciones de fabricación.}

Costos por órdenes de fabricación. - También conocido con los nombres de costo por órdenes específicas de producción, por lotes de trabajo o por pedidos de los clientes.

Costos por Procesos. - Este sistema se utiliza en aquellas empresas cuya producción es uniforme y el patrón de producción es repetitivo o continuo.

\section{Componentes de costos}

Entre los componentes de costo es común encontrar en cada una de las definiciones revisadas (Reyes, s.f.) (Bonilla y Prada, 1999) (Gómez, 1999).

El consumo de materia prima, los salarios, prestaciones, aportes patronales aplicables a:

La mano de obra directa, del personal de apoyo, administración o control de la producción, Otros cargos hechos al departamento de operaciones o producción, como materiales indirectos, depreciación y mantenimiento de las instalaciones o equipos de producción, seguros, energía, servicio de acueducto y teléfono. 
Según los aspectos económicos involucrados en la toma de decisiones

Los costos bajo este aspecto adquieren un sentido diferente y denominaciones diversas tales como:

Costos Incrementales. - Son costos adicionales en los que no se incurriría si no se emprende un determinado proyecto.

También pueden considerarse como la diferencia en costos totales que resulta de un cambio.

Costos de Oportunidad o Imputados: Son costos hipotéticos en el sentido que son ajenos a la contabilidad o no reconocidos como tales, en este sentido se dice que son costos teóricos y de gran importancia en la toma de decisiones financieras.

Costos aplazables. - Son aquellos costos que pueden ser desplazados hacia el futuro con poco o casi ningún efecto sobre la eficiencia de las operaciones corrientes.

Costos evitables e inevitables. - Se les conoce como costos vivos y costos extinguidos respectivamente. Los vivos son aquellos que representan erogaciones en el futuro, de ahí que son los únicos costos relevantes para analizar una decisión a corto plazo.

Los costos inevitables o extinguidos representan recursos económicos que han sido comprometidos y no se pueden recuperar.

Costos Controlables y no Controlables. - Son aquellos que están directamente influenciados por un ejecutivo dentro de un lapso de tiempo definido. (Aizaga, 2016)

\section{Contabilidad de costos}

\section{Definición}

Es un sistema de información por el cual se determina el costo incurrido al realizar un proceso productivo y la forma como se genera este, en cada una de las actividades en la que se desarrolla la producción. Se entiende por costo la suma de las erogaciones en la que incurre una persona para la adquisición de un bien o servicio, con la intención que gen ere un ingreso a futuro. (Medina, 2007) 
La Contabilidad de Costos es un sistema de información diseñado para suministrar información a los administradores de los entes económicos, comerciales industriales y de servicios que les brinda la oportunidad de planear, clasificar, controlar, analizar e interpretar el costo de bienes y servicios que produce o comercializa. (Anónimo, 2017)

La contabilidad de costos mide, analiza y reporta información financiera y no financiera relacionada con los costos de adquisición o uso de los recursos dentro de una organización. La contabilidad de costos moderna parte de la perspectiva de que la recolección de la información de costos está en función de las decisiones gerenciales que se tomen.

"La contabilidad es una herramienta valiosa en la toma de decisiones. Para que esta técnica sea aún más trascendente en las empresas, conviene generar y mantener una especialización que se encargue de la determinación del costo de hacer un bien o generar un servicio. El uso que se da a los costos es realmente importante porque se convierte en una fuente de datos pasados y presentes, sobre los cuales se pueden tomar decisiones, ayuda al control de los recursos y facilita la planificación."

"El costo de producir se entenderá como el valor monetario que se invierte en elementos materiales, fuerza laboral necesaria y demás insumos requeridos para fabricar bienes o sus partes y procesos, así como para generar servicios o productos intangibles cuya presencia beneficiosa es incuestionable con el objeto de obtener un producto o un servicio.

\section{Importancia de la Contabilidad de Costos}

"La determinación del costo es también importante para mejorar o mantener la imagen ante los clientes, quienes perciben desde afuera las fortalezas o debilidades administrativas ya que, con frecuencia, las empresas que carecen de un sistema apropiado de costos no son consistentes en sus precios ni en sus negociaciones y, especialmente no pueden sustentar con cifras confiables un aumento de tarifas, como el caso del aumento de precio del transporte público o de las tarifas eléctricas o telefónicas, aunque últimamente se han hecho esfuerzos para implementar un sistema de costeo apropiado en ellas." 
"Por lo tanto, la contabilidad de costos tiene su ámbito natural en las empresas industriales y las de servicios especializados, pues esta técnica de registro se encarga de llevar el detalle de todo cuanto sucede o deja de suceder en el sector productivo de toda industria." (Villalba Benavides, 2016)

\section{Actividades básicas de la contabilidad de costos}

La contabilidad de costos debe desarrollar cuatro actividades básicas para satisfacer sus objetivos:

Primera: Medición del costo. Acumulación de información necesaria para determinar el costo final de un producto: materiales, mano de obra, otros insumos, etc.

Segunda: Registro de los costos en los libros de contabilidad.

Tercera: Análisis de los costos para diferentes propósitos: planeación de operaciones, identificación de tendencias, etc.

Cuarto: Presentación detallada de informes para la toma de decisiones internas. (Lemus, 2010)

\section{Clasificación de costos en la contabilidad}

Como la contabilidad de costos tiene dentro de sus objetivos obtener la información necesaria para controlar la producción, planear las actividades de una empresa y tomar decisiones:

Por lo tanto, se clasifican en:

\section{De acuerdo a la función empresarial. - La cual se pueden dividir en dos:}

Costos de manufacturas o de producción. - Propios de las empresas que transforman materia prima en productos terminados.

Costos de mercado. - Cuya finalidad es calcular lo que cuesta distribuir un producto.

\section{Según la naturaleza de las operaciones de fabricación. - Los costos se dividen en:}

Costos por orden de fabricación. - Propios de empresas que elaboran sus productos.

Costos por procesos. - Utilizados en empresas de producción masiva y continúa utilizados en artículos similares. 


\section{De acuerdo al periodo en que fueron calculados:}

Costos históricos. - En las cuales en la gran mayoría de los datos son reales y se van presentando durante el periodo contable.

Costos predeterminados. - Los cuales se estiman a través de la experiencia, utilizando registros estadísticos y se lo utiliza para elaborar y controlar el presupuesto maestro.

\section{De acuerdo a la actividad. - Se dividen en:}

Costos directos. - Los cuales se pueden identificar de una manera directa a una actividad, como la materia prima del producto.

Costos indirectos. - Estos no se pueden llevar de una manera directa una actividad, hay que distribuirlo a una base equitativa.

\section{De acuerdo a la productividad. - Los costos pueden ser:}

Costos de la inefectividad. - Aquellos causado por no hacer el trabajo correctamente.

Costos de ineficiencia. - Se refiere a fallas y defectos detectados después

de expedir el producto o servicio al cliente interno o externo.

\section{Análisis de utilidades}

El análisis de utilidades a la luz del comportamiento de los costos y de los ingresos, es un factor masivo para la dirección de cualquiera empresa en sus políticas de administración, más aún en condiciones especiales como la reducción de volumen de ventas, escasez de materiales y demandas anormalmente elevados o reducidas. Los cambios en las utilidades se generan básicamente de dos maneras:

Cambios en los ingresos

Cambio en los costos

Para producción únicas los cambios en los ingresos y en los costos serán consecuencia de dos factores, precio y el volumen. Para producciones derivadas las utilidades se verán afectadas además de la mezcla de productos. (Moncada, 2008) 
Elementos del costo

Materia prima

Las definiciones sobre materia prima (materiales) son varias debido a que son la parte primordial en la elaboración del producto además de ser el primer elemento del mismo y ser una parte tangible dentro de la elaboración y la presentación del producto final.

De esta manera (Polimeni, Fabozzi, \& Adelberg, 1994) menciona que materiales "son los principales recursos que se usan en la producción; estos se transforman en bienes terminados con la adición de mano de obra directa y costos indirectos de fabricación.”

(Horngren, 2010) menciona por otro lado que "se incluyen los de adquisición de todos los materiales que, en última instancia, se convertirán en parte del objeto de costos (productos en elaboración y luego productos terminados), y que se pueden atribuir al objeto de costos de una manera económicamente factible". Esto en cuanto tiene que ver a costos adicionales por adquisición de materia prima como fletes e impuestos en importaciones como los aranceles.

\section{La materia prima está clasificada en dos grupos:}

Materia prima directa por su fácil identificación dentro del producto. - Son los que mayoritariamente representan el costo del producto y por lo tanto son la base fundamental en la producción de un producto. Ejemplo: En la elaboración de un comedor la materia prima directa es la madera.

Materia prima indirecta. - Son los materiales que se usan en la producción de un producto, pero no son materiales directos ejemplo: En la elaboración del ismo comedor mencionado anteriormente la materia prima indirecta es la cola o pega, tornillos.

\section{Mano de obra}

Se define según (Polimeni, Fabozzi, \& Adelberg, 1994) mano de obra como "el esfuerzo físico o mental empleados en la fabricación de un producto". (p. 12) De acuerdo a (Gómez, 2005) menciona dentro de naturaleza de mano de obra aspectos importantes como: "El pago de los trabajadores y demás personas que laboran en una empresa constituye la mano de obra, cuya incidencia sobre la producción, ya sea directa o indirecta, es notoria desde todo punto de vista. 
Gracias a la mano de obra, en su acción sobre equipos y maquinas, los materiales se convierten en partes especificas o en productos terminados."

\section{El costo de la mano de obra por lo tanto se convierte en el segundo elemento del costo así también se clasifica en:}

La mano de obra directa. - Es según (Polimeni, Fabozzi, \& Adelberg,

1994) "la que está directamente involucrada en la fabricación de un producto terminado que puede asociarse con este con facilidad y que representa un importante costo de mano de obra en la elaboración del producto."

La mano de obra indirecta. - Es la mano de obra que no se considera como mano de obra directa pero que si está incluida en la fabricación de un producto y al mismo tiempo se incluye como costo indirecto de fabricación.

\section{Costos indirectos de fabricación}

"Los costos indirectos de fabricación, debido a que constituyen un elemento indirecto del costo del producto, no pueden asociarse o cargarse con facilidad a una orden de trabajo o departamento específico; son costos que se aplican a la producción, puesto que ocurren de forma no uniforme, dando origen a la realización de estimaciones."

\section{La división más generalizada de los costos indirectos de fabricación es la siguiente:}

"Materiales indirectos. - Hacen parte de los materiales indirectos los combustibles, los lubricantes, las herramientas de poco valor y los suministros de fábrica, como tornillos o pegamentos."

"Mano de obra indirecta. - Se catalogan dentro de este renglón los siguientes costos: salarios de empleados de oficinas de administración de fábrica, cronometradores, empleados de superintendencia, empleados de almacén y ayudantes, conductores, fogoneros, primas por horas extras, tiempo ocioso, salario de capataces, etcétera."

"Otros costos indirectos. - Dentro de este renglón figuran depreciación (de edificios y maquinas), impuestos, seguros, alquileres, servicios públicos (agua, luz, teléfono) repuestos de maquinaria, prestaciones sociales de los obreros de producción indirecta, costos de fleten en compra de 
materiales, conservación de edificios, de maquinaria, de muebles y enseres. De equipo y automotores, etcétera.” (Villalba Benavides, 2016)

\section{Elementos del costo de producción}

Se trata de aquellos elementos indispensables para producir un artículo o prestar un servicio:

Materiales directos. - En la fabricación de un artículo, interviene diversos materiales, aquellos que realmente forman parte integral del producto terminado y que cumplen con las características de:

Valor: tiene un valor significativo.

Uso: uso relevante dentro del producto.

Son denominados materiales directos, y se consideran como el primer elemento del costo de producción.

Mano de obra directa. - Es la remuneración (salario, prestaciones sociales y aportes patronales) a que se hacen acreedores los trabajadores que intervienen directamente en la fabricación de los productos, por el tiempo realmente trabajado ya sea manualmente o mediante el accionamiento de máquinas encargadas de la transformación de materias primas y demás materiales en producto terminado.

Costos indirectos de fabricación (CIF). - Constituidos por los materiales indirectos, la mano de obra indirecta, y aquellas erogaciones o desembolso de valores indispensables para suplir algunos requerimientos propios del proceso productivo, tales como servicios públicos, alquiler de planta, arrendamiento de oficinas de producción, seguros de planta, entre otros.

\section{Sistemas de costeo}

Para determinar el costo de un producto, existen varias metodologías que se utilizan según el proceso productivo empleado en la empresa:

Costos por órdenes de producción: Conocido también como sistema de costos por lotes o por pedidos específicos. Los costos se acumulan en cada orden de producción por separado y la 
obtención de los costos unitarios es cuestión de una simple división de los totales correspondientes a cada orden, por el número de unidades producidas en esta.

Costos por procesos. - Es apto para empresas cuyas condiciones de producción no sufren cambios significativos, producen una sola línea de artículos, o fabrican productos muy homogéneos, en forma masiva o continua, cumpliendo etapas sucesivas (procesos) hasta su terminación total. En este sistema la unidad de costeo es el proceso de producción, acumulándose los costos por cada uno de estos durante un período de tiempo determinado.

Costos $\mathrm{ABC}$ o Costeo Basado en Actividades. - Es una metodología que mide el costo y el desempeño de actividades, recursos y objetos de costo. Los recursos se asignan primero a las actividades; después los costos de las actividades se asignan a los objetos de costo según el uso.

\section{Costos indirectos de fabricación}

Se llama así, al conjunto de costos de fábrica que intervienen en el proceso productivo y no se identifican como Material Directo ni Mano de Obra Directa, pudiéndose referir a los siguientes conceptos:

Material indirecto. - Todo aquel material que forma parte integral del producto, pero su valor no es representativo respecto al costo por unidad.

Mano de obra indirecta. - Son los salarios, prestaciones y aportes patronales a que den lugar todos los trabajadores de la fábrica cuya actividad está relacionada con el proceso productivo y aquellos considerados como mano de obra directa (operarios de producción) por su labor no productiva.

Suministros de fábrica. - Están representados por todo tipo de materiales consumidos por la planta de producción, como pueden ser papelería, jabones, aceites y grasas lubricantes, etc.

Consumos de fábrica. - Corresponde a este rubro, todos aquellos costos que se refieren a diversos servicios como arrendamiento, teléfono, servicios públicos, mantenimiento, depreciación, etc., siempre que estén relacionados con la planta fabril directamente. 


\section{Departamentalización}

En la planta fabril de una empresa existen diferentes departamentos que se pueden dividir en dos grandes grupos:

Departamentos de Producción también llamados Centros de Costos de Producción (CCP). - Son aquellos centros de producción donde hombres y equipos realizan una actividad común, formando parte del proceso productivo. Estos departamentos incurren en los costos de materiales directos y de mano de obra directa, pueden tener C.I.F. algunos de estos son directos y otros indirectos. Los departamentos de producción son los únicos que incurren en costos primos.

Departamentos auxiliares o Centros de Costos Auxiliares (CCA). - Son aquellos departamentos cuyos servicios los utilizan los departamentos principales o de producción, de ahí el reparto de los costos a los departamentos de producción. Estos departamentos auxiliares pueden surgir según las necesidades que consideren las directivas y ellos incurren solo en costos indirectos de fabricación.

A continuación, se presenta una lista de departamentos auxiliares que pueden existir en una fábrica:

Ingeniería Industrial.

Control de Calidad.

Productividad y Competitividad.

Servicios Generales.

Mantenimiento de Fábrica.

Supervisión.

Restaurante.

Administración de Producción.

Servicios asistenciales.

Personal. 
Administración de Material.

Generación de Energía.

Control de Producción.

Mantenimiento de Maquinaria y de planta.

Contabilidad de Fábrica

Los C.I.F. se dividen con respecto al presupuesto por departamentos en dos tipos:

Costos indirectos de fabricación directos. - Son aquellos cuyo presupuesto se puede hacer por cada departamento de producción o auxiliar de una manera directa, así como también sus costos reales se identifican y utilizan en cada departamento sin tener que efectuar reparticiones. Se consideran a continuación los siguientes costos indirectos de fabricación directos:

Herramientas.

Combustible.

Salarios de jefes de departamento.

Salarios de supervisores.

Mano de obra indirecta.

Repuestos.

Fletes de compras de materiales.

Materiales indirectos.

Prestaciones sociales de la mano de obra indirecta.

Abastecimiento restaurante.

Tiempo no productivo.

Costos indirectos de fabricación indirectos. - Se pueden conocer para toda la fábrica, pero no se pueden identificar para cada departamento por lo tanto se denominan Indirectos. Por ejemplo, el 
consumo de energía eléctrica se puede conocer este costo para toda la fábrica, pero no se puede identificar para cada departamento. Otros serian:

\section{Teléfono.}

Agua.

\section{Electricidad.}

Depreciación de edificios.

Depreciación maquinaria.

Seguros.

Impuestos prediales

Procedimientos para la distribución de los CIF

Los C.I.F. son complejos en su control y distribución a los respectivos departamentos. El problema de distribución de C.I.F. se puede resumir en cuatro pasos.

Paso 1: Identificar y acumular todos los costos indirectos de fabricación del periodo. - Consiste en tomar cada uno de los costos causados en el periodo y soportados en documentos.

Paso 2: Identificar los C.I.F. que le corresponden a cada departamento de producción o auxiliar. A este procedimiento se conoce como distribución primaria: los costos identificados se distribuyen en cada departamento según sea su consumo, de acuerdo a una base apropiada.

Paso 3: Posteriormente los departamentos auxiliares deben ser absorbidos por los departamentos de producción. - Repartiendo sus costos mediante una base que guarde relación directa entre los departamentos de auxiliares y los de producción.

Paso 4. Finalmente se distribuyen los valores acumulados en cada CCP entre las unidades procesadas de acuerdo al sistema de costeo utilizado. - Llamado distribución final. (Arias Montoya, Portilla, \& Fernández, 2010) 
Es importante destacar que el control interno no solo abarca el entorno financiero y contable sino también los controles cuya meta es la eficiencia administrativa y operativa dentro de la organización empresarial, industrial, entre otras; salvaguardando los procesos internos de datos sobre el costo de producir la estructura del costo, el beneficio que les brinda a cada una de las líneas de producción y ayuda al control de los elementos del costo y a quienes operan sobre ellos.

"El control interno es diseñado e implementado por la administración para tratar los riesgos de negocio y de fraude identificados que amenazan el logro de los objetivos establecidos, como la confiabilidad de la información financiera.

El control interno es el que desarrolla dentro de una empresa por el interés de la administración, porque desea asegurar al máximo la conducción ordenada y eficiente de todas las actividades de la misma, utilizando como herramienta procedimientos o forma prestablecidas de control que aseguren su estructura interna y comportamiento, además debe contar con personal idóneo paras funciones a cumplir”. (María, 2014)

El control interno es el medio para alcanzar un fin, estableciendo normativas al personal que conducen en todos los niveles, también hace referencia a la organización y procedimiento interno y externo, no solo se trata de manuales administrativos o de funciones sino de evolución a los sistemas en donde se llevan los procesos diarios para la seguridad total, manifestando a una cadena de actividades que pone en marcha la producción de un bien y servicios derivados de la materia prima.

Con la aplicación del control interno en la cooperativa de producción agropecuaria de Chone garantizara el desarrollo y crecimiento de los objetivos proyectados enlazando cada una de las áreas que conforman la cooperativa logrando la competitiva en el mercado, un control interno es importante en esta compañía le permitirá a la gerencia obtener información financiera eficiente y sirva de instrumento en la toma de decisiones reflejando la situación económica actual bajo estándares contables.

Por ende, comprende el plan de organización en todos los procedimientos coordinados de manera coherente a las necesidades del negocio, para proteger y resguardar sus activos, verificar su exactitud y confiabilidad de los datos contables. 
Mediante las técnicas utilizadas, entrevista y la encuesta aplicada al personal, se determinará la hipótesis trazada, la misma que comprueba si beneficia o no a la Cooperativa.

H1: La evaluación del sistema de Control Interno contable permitirá mejorar los procesos en los costos de fabricación de los productos lácteos en el departamento contable de la Cooperativa De Producción Agropecuaria Chone Ltda.

H0: La evaluación del sistema Control Interno contable no permitirá mejorar los procesos en los costos de fabricación de los productos lácteos en el departamento contable de la Cooperativa De Producción Agropecuaria Chone Ltda.

La variable independiente se determina control interno y la variable dependiente se refiere a los costos de fabricación, la cual de describen las siguientes tareas científica, para alcanzar el propósito de la investigación:

Analizar desde el punto de vista histórico el movimiento en cuánto a los costos y gastos en la elaboración de productos lácteos en la Cooperativa De Producción Agropecuaria Chone Ltda.

Valorar los fundamentos teóricos relacionados con el registro de costos y gastos invertido para la elaboración de los productos lácteos en la Cooperativa De Producción Agropecuaria Chone Ltda.

Determinar la situación actual económica que adquiere la cooperativa por los productos lácteos vendidos.

Diseñar un manual de evaluación a los sistemas de control en la producción de lácteos en la Cooperativa De Producción Agropecuaria Chone Ltda. para mejorar los procesos internos.

Para desarrollar la investigación y alcanzar el objetivo establecido anteriormente se consideró la máxima autoridad como responsable de la Cooperativa y todo el personal que la conforma, utilizando los métodos de análisis y síntesis obtenido información pertinente evaluando las características establecida principales del problema planteado y así verificar el rendimiento laboral de los subordinados y la situación actual económica que presenta la Cooperativa mediante documentación previa y realizar el debido análisis. 
Mediante el método de inducción se planteó la hipótesis y con la metodología de deducción podremos comprobar que con la aplicación de un control interno podrá la Cooperativa de Producción Agropecuaria Chone Ltda mejorar los procesos de los costos de fabricación de los productos lácteos mediante la elaboración de un informe costos y gastos.

Se aplicó como herramienta de apoyo las técnicas básicas de recolección de datos para obtener la información pertinente fue la entrevista que se la aplico al Ingeniero Tulio Arteaga como responsable de la Cooperativa Agropecuaria de Chone y la encuesta aplicada al personal que la conforma.

\section{Importancia del Problema}

El problema científico de la presente investigación es la ineficiencia de un manual de procedimiento aplicable al sistema de control interno en la Cooperativa de Producción Agropecuaria Chone Ltda. para prevenir riesgo futuro y alcanzar el objetivo establecido.

El objetivo general es realizar un control interno en el proceso de los costos de fabricación en la elaboración de productos lácteos en la Cooperativa De Producción Agropecuaria "Chone Ltda." que permitirá establecer un mejor registro en los procesos de los costos de producción. Mediante las técnicas utilizadas, entrevista y la encuesta aplicada al personal, se determinará la hipótesis trazada, la misma que comprueba si beneficia o no a la Cooperativa.

El control interno en los costos de fabricación de los productos lácteos en la Cooperativa de Producción Agropecuaria "Chone Ltda" es de vital importancia porque:

Establece medidas para corregir las actividades, de tal forma que se alcancen planes exitosamente.

Se aplica a todo (cosas, personas y a los actos.)

Determina y analiza rápidamente las causas que pueden originar desviaciones, para que no se vuelvan a presentar en el futuro.

Localiza a los lectores responsables de la administración, desde el momento que se establecen medidas correctivas. 
Proporciona información acerca de la situación de la ejecución de los planes, sirviendo como fundamento al reiniciarse el proceso de planeación.

\section{Reduce costos y ahorra tiempo al evitar errores.}

$\mathrm{Su}$ aplicación incide directamente en la racionalización de la administración y consecuentemente, en el logro de la productividad de todos los recursos de la empresa. (Quiroz, 2011)

\section{Metodología}

Para desarrollar la investigación y alcanzar el objetivo establecido, se utilizó los métodos de análisis y síntesis, para obtener información pertinente y evaluar las características establecidas del problema planteado y así verificar el rendimiento laboral de los subordinados y la situación actual económica que presenta la institución mediante documentación previa y su debido análisis.

Mediante el método de inducción se planteó la hipótesis y con el método de deducción se comprueba que con la aplicación de un control interno podrá la Cooperativa de Producción Agropecuaria Chone Ltda mejorar los procesos de los costos de fabricación de los productos lácteos mediante la elaboración de un informe costos y gastos.

Se aplicó como herramienta de apoyo las técnicas básicas de recolección de datos para obtener la información pertinente como la entrevista que se la aplico al Ingeniero Tulio Arteaga como responsable de la Cooperativa Agropecuaria de Chone y la encuesta aplicada al personal que la conforma.

\section{Resultados esperados}

Los resultados que se desean obtener en esta investigación es esencialmente para mejorar los procesos de la empresa realizados por los directivos y personal administrativo como se detalla a continuación:

Beneficiar al gerente general y subgerente de la cooperativa de producción agropecuaria Chone Ltda. sistematizando los procesos productivos dado en el sistema. 
Facilitar el incremento de utilidad cumpliendo a cabalidad los procedimientos establecidos en el manual.

Adquirir información eficaz y eficiente de los recursos obtenidos para la toma de decisiones.

Promover la buena práctica de las normativas vigentes reduciendo impacto socio económico Contribuir al desarrollo de tareas específicas generales seleccionadas en el manual para llevar un mejor control interno en el sistema

\section{Conclusiones}

Al terminar la investigación realizada en la cooperativa de producción agropecuaria Chone Ltda. se concluye:

En el estudio realizado en la cooperativa de producción agropecuaria Chone Ltda. se detecta la inexistencia de un manual de procedimiento aplicable al sistema de control interno contable, para determinar los procesos productivos en la realización o transformación de productos.

Con las técnicas aplicables se determinó que en la cooperativa existe una serie de fichas para llevar un registro de los materiales e insumos para obtener un mejor control, pero estas no son utilizadas correctamente, por el personal que labora en la institución.

\section{Referencias Bibliográficas}

Aizaga, F. L. (2016). Estado del Arte de la Contabilidad de Costos. Scopus, 7-10.

Anónimo. (2017). zenempresarial.files.wordpress.com. Obtenido de https://zenempresarial.files.wordpress.com/2009/12/contabilidad-de- costos.pdf

Arias Montoya, L., Portilla, L., \& Fernández. (2010). La Distribución de Costos Indirectos de Fabricación, Factor Clave al Costear. Redalyc, 79-82. Obtenido de http://www.redalyc.org/html/849/84917249014/

Barrios, J. P. (8 de Septiembre de 2014). bitstream. Obtenido de http://190.242.62.234:8080/jspui/bitstream/11227/2130/1/TESIS\%20CONTROL\%20INTERNOPRESENTAC ION\%20F INAL.pdf 
Gaitan, R. E. (2015). Control Interno y fraudes Análisis de informe COSO I, II y III con base en los ciclos transaccionales. Bogotá: Tercera Edición.

Jr. Camino Carrillo, J. M. (Agosto de 2014). Marco_Conceptual_Control_Interno_CGR.pdf. Obtenido http://doc.contraloria.gob.pe/Control- Interno/web/documentos/Publicaciones/Ma rco_Conceptual_Control_Interno_CGR.pdf

Lemus, W. J. (2010). Contabilidad de Costos. Bogotá Colombia: Segunda Edición.

Obtenido de http://www.sanmateo.edu.co/documentos/publicacion- contabilidad-costos.pdf

María, D. M. (24 de Enero de 2014). repo.uta.edu.ec. Obtenido de http://repo.uta.edu.ec

Medina, R. A. (2007). Sistemas de Costos-Un proceso para su implementación.

Colombia: Primera Edición. Obtenido de https://es.slideshare.net/jacosol/libro-de -contabilidadde-costos Moncada, A. M. (Abril de 2008). repositorio.utp.edu.co. Obtenido de http://repositorio.utp.edu.co/dspace/bitstream/handle/11059/901/6581552M516.pdf;jsessionid=5 A2023B97E064CCF35BC00A3E812802C?sequ ence=1

Nagua Cartuche, M. V. (2016). El control interno en las PYMES familiares, una oportunidad para mejorar la competitividad empresarial. Scielo, 3-4. Obtenido de http://repositorio.utmachala.edu.ec

Orozco, M. y. (2013). Costos de Producción. Scielo, 15-19.

Quiroz, B. J. (7 de Mayo de 2011). Obtenido de http://dspace.utpl.edu.ec/bitstream/123456789/6798/1/Tesis\%20Salas\%

O 20Quiroz\%20Blanca.pdf

Quiroz, B. J. (7 de Mayo de 2011). dspace.utpl.edu.ec. Obtenido de http://dspace.utpl.edu.ec/bitstream/123456789/6798/1/Tesis\%20Salas\%20Quiroz\%20Blanca.pdf

Quiroz, B. J. (07 de Mayo de 2011). https://www.google.com.ec/search?bi $\mathrm{W}=1360 \& \mathrm{bih}=662 \& \mathrm{q}=$ concepto $+\mathrm{de}+\mathrm{control}+$ interno + segun + autores $\&$ oq $=$ control + interno + pdf $\&$ gs_l=psy-

ab.1.2.0i71k114.0.0.0.8096.0.0.0.0.0.0.0.0..0.0...0...1..64.psyab..0.0.0...0.MV4txvIGkk8. 
Obtenido

de:

http://dspace.utpl.edu.ec/bitstream/123456789/6798/1/Tesis\%20Salas\%20Quiroz\%20Blanca.pdf

Rivas Márquez, G. (2011). Modelos contemporáneos de control interno. Fundamentos teóricos.

Redalyc, 117-125. Obtenido de http://www.redalyc.org/pdf/2190/219022148007.pdf

Rodrigo. (2006). Control Interno. En E. Gaitan, Control Interno y fraudes con base los ciclos transaccionales: Análisis del Informe COSO I y II (pág. O 19). Bogotá: 2a Ediciones. Obtenido dehttps://dspace.ups.edu.ec/bitstream/123456789/1450/1/CAP ITULO\%201.pdf

Villalba Benavides, M. E. (2016). Los costos de producción y la rentabilidad en la elaboración de muebles en el Centro Artesanal Huambaló durante el período 2015. Scopus, 23-29. Obtenido de http://repositorio.uta.edu.ec/jspui/handle/123456789/23843

\section{Anexos}

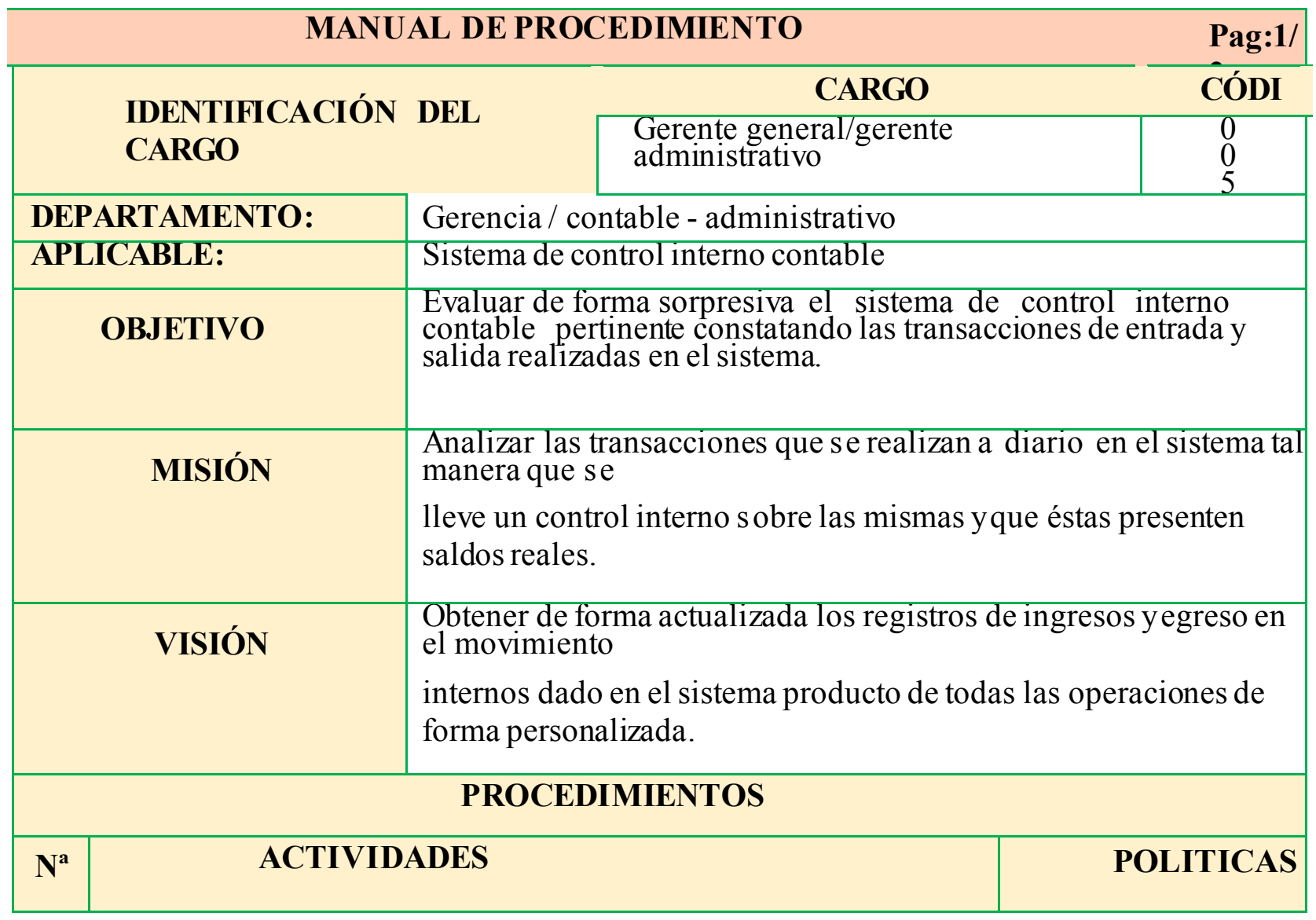


Susy T. Toala Mendoza, Karina C. Arteaga Muñoz, Jhon A. Álava Intriago

\begin{tabular}{|c|c|c|c|}
\hline \multirow{7}{*}{1} & \multicolumn{2}{|r|}{$\begin{array}{l}\text { Examinar el sistema de control verificando si se realzan los } \\
\text { registros } \\
\text { de todos los materiales e insumos de ingreso y egreso }\end{array}$} & $\begin{array}{l}\text { Norma de control } \\
\text { interno }\end{array}$ \\
\hline & $\mathbf{N}^{\mathrm{a}}$ & ACCIONES & \\
\hline & 1 & Verificar las transacciones de entrada y salida en su totalidad & 600seguimiento \\
\hline & 2 & Comprobar que el funcionamiento en el sistemas que cumpla & \multirow{3}{*}{$\begin{array}{l}\text { La máxima } \\
\text { autoridad y } \\
\text { directivos do } \\
\text { entidad, establecerán }\end{array}$} \\
\hline & 3 & $\begin{array}{l}\text { Detectar que el sistema es té siendo utilidad con fines } \\
\text { lucrativo para la Cooperativa }\end{array}$ & \\
\hline & 4 & $\begin{array}{l}\text { Constatar si los materiales e insumos no ente con costos } \\
\text { alterados }\end{array}$ & \\
\hline & 5 & $\begin{array}{l}\text { Verificar si la cantidad de producto es idóneo con la cantidad } \\
\text { de materiales utilizado en la producción. }\end{array}$ & $\begin{array}{l}\text { procedimientos de } \\
\text { seguimiento }\end{array}$ \\
\hline \multirow{7}{*}{2} & \multicolumn{2}{|r|}{$\begin{array}{l}\text { Analizar el estado económico de la entidad arrogado por el } \\
\text { sistema de } \\
\text { control interno }\end{array}$} & \multirow{4}{*}{ 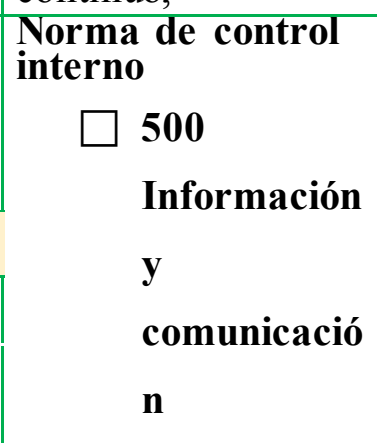 } \\
\hline & $\mathbf{N}^{\mathbf{a}}$ & ACCIONES & \\
\hline & 1 & Hacer comparaciones de un periodo a otro. & \\
\hline & 2 & $\begin{array}{l}\text { Constatar los costos históricos y reales de materiales e } \\
\text { insumos }\end{array}$ & \\
\hline & 3 & $\begin{array}{l}\text { Verificar los ingresos obtenido en el mes y los gastos } \\
\text { generados } \\
\text { derivado de la misma }\end{array}$ & \multirow{2}{*}{$\begin{array}{l}\text { Los sistemas de } \\
\text { información contarán } \\
\text { con }\end{array}$} \\
\hline & 4 & Comparar si los ingresos superan a la inversión realizada. & \\
\hline & 5 & $\begin{array}{l}\text { Comprobar s i los datos obtenido en él estado económico son } \\
\text { reales. }\end{array}$ & $\begin{array}{l}\text { adecuados para } \\
\text { garantizar } \\
\text { confiabilidad, } \quad \mathrm{S}\end{array}$ \\
\hline
\end{tabular}

\section{MANUAL DE PROCEDIMIENTO}

IDENTIFICACIÓN DEL CARGO

\begin{tabular}{l|l}
\hline DEPARTAMENTO: & Gerencia / contable - administrativo \\
\hline APLICABLE: & Sistema de control interno contable
\end{tabular}

\section{CARGO}

Gerente general/gerente administrativo 


\begin{tabular}{|c|c|c|c|c|}
\hline & \multicolumn{2}{|c|}{ OBJETIVO } & \multicolumn{2}{|c|}{$\begin{array}{l}\text { Evaluar de forma sorpresiva el sistema de control interno contable pertinente } \\
\text { constatando las transacciones de entrada y salida realizadas en el sistema. }\end{array}$} \\
\hline & \multicolumn{2}{|c|}{ MISION } & \multicolumn{2}{|c|}{$\begin{array}{l}\text { Analizar las transacciones que se realizan a diario en el sistema tal manera que } \\
\text { se lleve un control interno sobre las mismas y que éstas presenten saldos reales }\end{array}$} \\
\hline & \multicolumn{2}{|c|}{ VISIÓN } & \multicolumn{2}{|c|}{$\begin{array}{l}\text { Obtener de forma actualizada los registros de ingresos yegreso en el } \\
\text { movimiento } \\
\text { internos dado en el sistema producto de todas las operaciones de forma } \\
\text { personalizada. }\end{array}$} \\
\hline \multirow{7}{*}{3} & \multicolumn{2}{|c|}{$\mathbf{N}^{a}$} & ACCIONES & \multirow{7}{*}{$\begin{array}{l}\text { Interno Control } \\
\mathbf{6 0 0} \text { seguimiento } \\
\text { El seguimiento se } \\
\text { efectúa en forma } \\
\text { continua durante la } \\
\text { realización de las } \\
\text { actividades diarias en los } \\
\text { distintos niveles de la } \\
\text { entidad y a través de } \\
\text { evaluaciones periódicas } \\
\text { para } \\
\text { directamente la } \\
\text { efectividad de los } \\
\text { controles en un tiempo } \\
\text { determinado, también } \\
\text { puede } \\
\text { mediante la combinación } \\
\text { de las dos modalidades. }\end{array}$} \\
\hline & \multicolumn{3}{|c|}{ Mantener una adecuada supervisión y control de las actividades: } & \\
\hline & 1 & \multirow{2}{*}{\multicolumn{2}{|c|}{$\begin{array}{l}\text { Establecer fechas sorpresivas para la evaluación del control } \\
\text { Determinar el tiempo para evaluar la información pertinente } \\
\text { requerida por la misma. }\end{array}$}} & \\
\hline & 2 & & & \\
\hline & 3 & \multicolumn{2}{|c|}{$\begin{array}{l}\text { Realizar supervisión una vez al mes de forma sorpresiva para } \\
\text { controlar las actividades realizada en el sistema. }\end{array}$} & \\
\hline & 4 & \multicolumn{2}{|c|}{$\begin{array}{l}\text { Pedir informe detallado de un periodo dado y com probar si la } \\
\text { misma } \\
\text { es la realizado en el sistem a con forme lo estable el documento } \\
\text { físico. }\end{array}$} & \\
\hline & 5 & \multicolumn{2}{|c|}{$\begin{array}{l}\text { Supervisar los procesos de cada producto seleccionándolo } \\
\text { por periodo. }\end{array}$} & \\
\hline \multirow{5}{*}{4} & $\mathbf{N}$ & & ACCIONES & \multirow{5}{*}{$\begin{array}{l}\text { Norma de control } \\
\text { interno } \\
\begin{array}{l}\square 600-01 \\
\text { Seguimiento }\end{array}\end{array}$} \\
\hline & \multicolumn{3}{|c|}{$\begin{array}{l}\text { Verificar el informearchivo en físico tiene tener la misma estructura e } \\
\text { información redactado en el sis tema de control interno contable. }\end{array}$} & \\
\hline & 1 & \multicolumn{2}{|c|}{ Verificar fecha de realización. } & \\
\hline & 2 & \multicolumn{2}{|c|}{ Comprobar las firmas del documento físico. } & \\
\hline & 3 & \multicolumn{2}{|c|}{ Verificar si la utilidad arroja el mismo resultado en el sis tema. } & \\
\hline
\end{tabular}


Susy T. Toala Mendoza, Karina C. Arteaga Muñoz, Jhon A. Álava Intriago

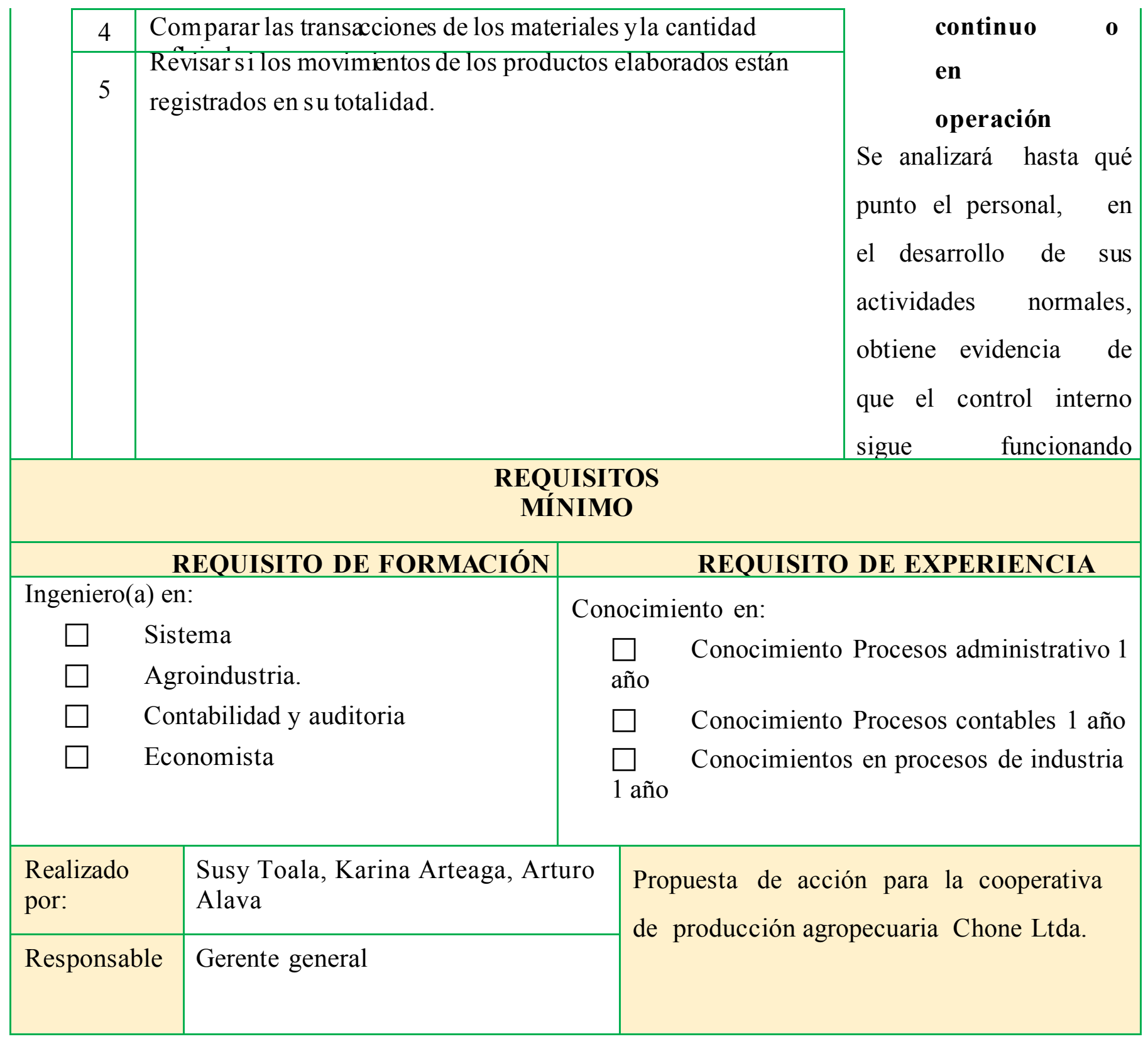

Fuente: Elaboración Propia 